OPEN ACCESS

Edited by:

Veerpal Singh Awana, National Physical Laboratory (CSIR),

India

Reviewed by:

Atsushi Fujimori,

The University of Tokyo, Japan Kenji Ishii,

National Institutes for Quantum Science and Technology, Japan

*Correspondence:

Matthias Hepting

hepting@fkf.mpg.de

Specialty section:

This article was submitted to Condensed Matter Physics,

a section of the journal

Frontiers in Physics

Received: 03 November 2021 Accepted: 30 November 2021

Published: 22 December 2021

Citation:

Hepting M, Dean MPM and Lee W-S (2021) Soft X-Ray

Spectroscopy of Low-

Valence Nickelates.

Front. Phys. 9:808683.

doi: 10.3389/fphy.2021.808683

\section{Soft X-Ray Spectroscopy of Low-Valence Nickelates}

\author{
Matthias Hepting ${ }^{1 *}$, Mark P. M. Dean ${ }^{2}$ and Wei-Sheng Lee ${ }^{3}$ \\ ${ }^{1}$ Max-Planck-Institute for Solid State Research, Stuttgart, Germany, ${ }^{2}$ Condensed Matter Physics and Materials Science \\ Department, Brookhaven National Laboratory, Ridge, NY, United States, ${ }^{3}$ Stanford Institute for Materials and Energy Sciences, \\ SLAC National Accelerator Laboratory and Stanford University, Menlo Park, CA, United States
}

Low-valence nickelates-including infinite-layer (IL) and trilayer (TL) compounds-are longstanding candidates for mimicking the high-temperature superconductivity of cuprates. A recent breakthrough in the field came with the discovery of superconductivity in hole-doped IL nickelates. Yet, the degree of similarity between low-valence nickelates and cuprates is the subject of a profound debate for which soft $\mathrm{x}$-ray spectroscopy experiments at the $\mathrm{Ni} L$ - and $\mathrm{O} K$-edge provided critical input. In this review, we will discuss the essential elements of the electronic structure of low-valance nickelates revealed by $\mathrm{x}$-ray absorption spectroscopy (XAS) and resonant inelastic $\mathrm{x}$-ray scattering (RIXS). Furthermore, we will review magnetic excitations observed in the RIXS spectra of IL and TL nickelates, which exhibit characteristics that are partly reminiscent of those of cuprates.

Keywords: infinite-layer nickelates, trilayer nickelates, low-valence nickelates, spectroscopy, RIXS, XAS, electronic structure, magnetic correlations

\section{INTRODUCTION}

Spectroscopy has proven to be a versatile tool for studying the charge, spin, lattice, and orbital degrees of freedom in quantum materials. Arguably, one of the most fascinating families of quantum materials are cuprate high-temperature superconductors [1-3]. Critical insights into cuprates have been provided by electron, neutron, and photon spectroscopy techniques-including photoemission and electron spectroscopy, scanning tunneling microscopy, optical and Raman spectroscopy, as well as inelastic x-ray and neutron scattering [4-10]. More recently, fresh perspectives on the enigmatic ground states of cuprates opened up especially due to resonant soft $\mathrm{x}$-ray spectroscopy studies. For instance, resonant inelastic x-ray scattering (RIXS) [11] revealed that damped spin excitations persist even for high hole-doping levels far away from the parent antiferromagnetically (AFM) ordered phase [12, 13]. Furthermore, RIXS allowed to probe the three-dimensional (3D) dispersion of low-energy plasmons [14-16], which arise due to the characteristic quasi-2D layered crystal structure of cuprates. Moreover, resonant elastic $\mathrm{x}$-ray scattering (REXS) and RIXS studies found that different types of static and dynamic charge orders emerge ubiquitously in cuprates [17-21]. Nevertheless, a comprehensive understanding of the most prominent phases in cuprates-including the pseudogap, strange metal phase, and superconductivity-remains elusive.

One approach to gain a deeper understanding of cuprates involves the targeted design of materials that mimic cuprate-typical properties, such as their layered quasi-2D crystal structure, $3 d^{9}$ electronic configuration, spin $S=1 / 2$ magnetic moments with antiferromagnetic (AFM) coupling, strong ligand-oxygen hybridization, and a lifted degeneracy of the active $e_{g}$ orbitals [22]. In principle, the discovery of superconductivity in a material that emulates at least a subset of 
these properties could allow to identify the hallmarks of cuprates that are crucial for invoking their exceptional hightemperature superconductivity.

In this context, long-standing candidates are Ni-based compounds, as $\mathrm{Ni}$ is a direct neighbor of $\mathrm{Cu}$ in the periodic table. In early works, it was speculated that doped $R E_{2} \mathrm{NiO}_{4}(R E=$ rare-earth ion), which is the $n=1$ member of the RuddlesdenPopper (RP) homologous series $R E_{n+1} \mathrm{Ni}_{n} \mathrm{O}_{3 n+1}$ [23], could become superconducting [24], as it is isostructural to the cuprate $\mathrm{La}_{2} \mathrm{CuO}_{4}$ and possesses similar charge and spin stripe ordered states [25]. The formal electronic configuration of $\mathrm{La}_{2} \mathrm{NiO}_{4}$, however, is $3 d^{8}\left(\mathrm{Ni}^{2+}\right)$ with $S=1[26,27]$, providing a possible rationalization for the observed absence of superconductivity. Perovskite nickelates $R_{E N i O}$ are the $n=$ $\infty$ member of the RP series with a formal $3 d^{7}\left(\mathrm{Ni}^{3+}\right)$ configuration and $S=1 / 2$, although $\mathrm{x}$-ray spectroscopic experiments indicated a $3 d^{8} \underline{L}$ configuration [28], with $\underline{L}$ denoting an $\mathrm{O}$ ligand hole. In their seminal work, Chaloupka and Khaliullin proposed a significant modification of the electronic structure of perovskite nickelates via tensile strain and incorporation into thin epitaxial heterostructures [29], breaking of the degeneracy of the $e_{g}$ orbitals, which in the extreme case could yield a half filled $3 d_{x^{2}-y^{2}}$ band and a single-sheet, cuprate-like Fermi surface [30]. In fact, spectroscopic studies detected new electronic and magnetic ground states in perovskite nickelate heterostructures, distinct from those of bulk nickelates [31-41]. Nevertheless, superconductivity has not been found to date [42-44], possibly due to the insufficient splitting of the orbital energy levels in the realized heterostructures [45, 46]. More recently, oxygen-reduced variants of the $n=3$ members of the RP series have attracted intense attention. These $R E_{4} \mathrm{Ni}_{3} \mathrm{O}_{8}$ trilayer (TL) nickelates are composed of three closely stacked square-planar $\mathrm{Ni}-\mathrm{O}$ layers with intervening $R E$ ions, which are separated by rocksalt type $R E-O$ blocking structures [47]. The electronic configuration of TL nickelates is $3 d^{8.67}$ per $\mathrm{Ni}$ on average $\left(\mathrm{Ni}^{1.33+}\right)$. It was found by $\mathrm{x}$-ray spectroscopy that metallic $\mathrm{Pr}_{4} \mathrm{Ni}_{3} \mathrm{O}_{8}$ exhibits low-spin configuration and a significantly lifted orbital degeneracy, suggesting a close analogy to cuprates [48]. Notably, the close parallel between TL nickelates and cuprates was further corroborates by RIXS, revealing the presence of strong AFM exchange coupling $J$ in $\mathrm{La}_{4} \mathrm{Ni}_{3} \mathrm{O}_{8}$ and $\mathrm{Pr}_{4} \mathrm{Ni}_{3} \mathrm{O}_{8}$ [49]. Nonetheless, superconductivity has not been detected in TL nickelates. This could be due to their electronic configuration, which corresponds to 1/3-hole-doping of a $\mathrm{Ni}^{1+}$ background and therewith falls into the overdoped regime of cuprates [48, 50, 51]. Experimental efforts to stabilize superconductivity via lowering the $\mathrm{Ni}^{1.33+}$ valence by electrondoping are ongoing. Along these lines, also the $n=5$ oxygenreduced $\mathrm{RP}$ variants $R E_{6} \mathrm{Ni}_{5} \mathrm{O}_{12}$ are promising candidates for superconductivity, as these quintuple layer nickelates exhibit a $3 d^{8.8}\left(\mathrm{Ni}^{1.2+}\right)$ configuration-analogous to optimally doped cuprates-already without additional electron-doping.

A breakthrough in the field came with the discovery of superconductivity in hole-doped nickelates with the IL crystal structure [52]. In more detail, epitaxial thin films of Sr- or Casubstituted $R E \mathrm{NiO}_{2}$ obtained via topotactic oxygen reduction of the perovskite phase show superconductivity below 9-15 K [52-58]. The parent compounds of these nickelates formally exhibit the $3 d^{9}\left(\mathrm{Ni}^{1+}\right)$ configuration with $S=1 / 2$, which qualifies them as isostructural and isoelectronic to the parent cuprates. Early neutron powder diffraction studies of parent IL nickelates, however, indicated absence of long-range AFM order $[59,60]$ and electrical transport measurements of films show weakly metallic behavior [61]. This is in stark contrast to parent cuprates, which are AFM Mott (charge-transfer) insulators [1]. Moreover, whereas first theoretical studies proposed that the electronic and magnetic correlations of IL nickelates and cuprates share close similarities [62], other theoretical works suggested significant distinctions, including a multiband character of nickelates [63]. Along these lines, insights from experiments can help to resolve the controversy about similarities and differences between IL nickelates and cuprates. In particular, recent $\mathrm{x}$-ray and electron energy-loss spectroscopic studies [64-66] unveiled a reduced $\mathrm{Ni}-\mathrm{O}$ hybridization, presence of a weakly interacting $R E 5 d$ metallic band, and overdamped spin excitations with a bandwidth as large as $200 \mathrm{meV}$ in IL nickelates.

In the following, we will review recent soft $\mathrm{x}$-ray absorption spectroscopy (XAS) and RIXS studies at the $\mathrm{O} K$-edge and $\mathrm{Ni} L$ edge of IL and TL nickelates. We will discuss the essential elements of their distinct electronic structure. Furthermore, the spin excitation spectra of IL and TL nickelates observed with RIXS will be reviewed.

\section{ELECTRONIC STRUCTURE}

$\mathrm{XAS}$ at the $\mathrm{O} K$-edge measures core-hole excitations from $\mathrm{O} 1 \mathrm{~s}$ to unoccupied $\mathrm{O} 2 p$ states and is also a sensitive probe of the covalent mixing between $\mathrm{O} 2 p$ and transition-metal $d$ states [67]. In particular the $\mathrm{O}-K$ pre-edge fine structure can provide valuable information about $\mathrm{Ni}-\mathrm{O}$ hybridized states and the associated electronic structure, for instance in the cases of $\mathrm{NiO}$ and perovskite $R E \mathrm{NiO}_{3}[28,67,68]$. In both materials, Ni $3 d$ orbitals strongly hybridize with oxygen ligands, giving rise to a pre-peak in the absorption spectra near the $\mathrm{O} K$-edge (Figure 1A). Due to different relative energy scales between the charge-transfer energy $\Delta$ and the Coulomb interaction $U$, according to the Zaanen-Sawatzky-Allen (ZSA) scheme [69], the former material falls into the regime of charge-transfer insulators, whereas the latter is a negative charge-transfer compound. In contrast, the $\mathrm{O} K$-edge absorption spectra of the IL nickelates $\mathrm{LaNiO}_{2}$ and $\mathrm{NdNiO}_{2}$ lack a prominent pre-edge peak (Figure 1A), suggesting a substantially weaker effective mixing between oxygen and the unoccupied $3 d$ states of the upper Hubbard band (UHB) of the $\mathrm{Ni}^{1+}$ cations [64]. In the case of cuprates, a prominent pre-peak feature is present in $\mathrm{O} K$-edge absorption spectra [70]. This is known to originate from the charge-transfer nature of these materials, with $\Delta$ smaller than $U$, and $\mathrm{O} 2 p$ states mixed with both the lower Hubbard band (LHB) and the UHB of the $\mathrm{Cu} 3 d_{x^{2}-y^{2}}$ states. A sizable pre-peak has also been observed in TL nickelates (Figure 1C), indicating mixing between $\mathrm{O} 2 p$ and the Ni UHB $[48,49]$. Upon hole-doping of cuprates, spectral weight shifts from the UHB pre-peak to a 

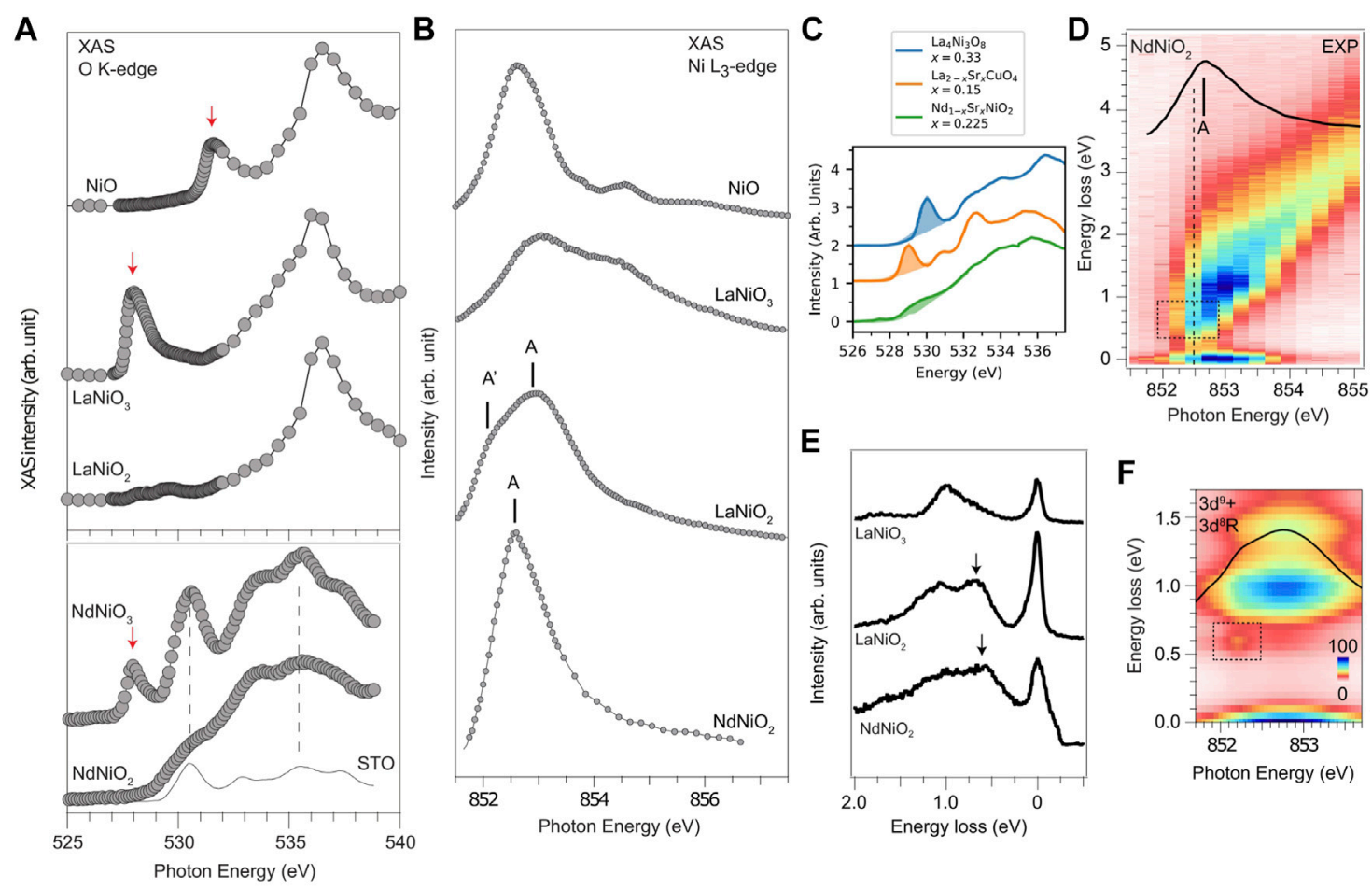

FIGURE 1 | Electronic structure of nickelates. (A) Upper panel: $\mathrm{O}$-edge XAS of $\mathrm{NiO}, \mathrm{LaNiO}_{3}$ and $\mathrm{LaNiO}_{2}$. Red arrows mark the pre-edge peaks indicative of $\mathrm{Ni}-\mathrm{O}$ hybridization. Lower panel: $\mathrm{O}$ K-edge XAS of $\mathrm{NdNiO}_{3}$ and $\mathrm{NaNiO}_{2}$. Dashed vertical lines indicate features of the $\mathrm{SrTiO}_{3}(\mathrm{STO}$ ) substrate (solid grey line) in the XAS spectra of $\mathrm{NdNiO}_{3}$ and $\mathrm{NdNiO}_{2}$ due to the film thickness being thinner than that of the La-based films in the upper panel. Spectra are vertically offset for clarity. (B) $\mathrm{Ni} L_{3}$-edge $X A S$ of $\mathrm{NiO}, \mathrm{LaNiO}_{3}, \mathrm{LaNiO}_{2}$, and $\mathrm{NaNiO}_{2}$. The $\mathrm{La} \mathrm{M}_{4}$-line was subtracted from the $\mathrm{LaNiO}_{3}$ and $\mathrm{LaNiO}_{2}$ spectra. (C) Comparison of the O K-edge pre-peak intensities (shaded areas) of a TL nickelate $\left(\mathrm{La}_{4} \mathrm{Ni}_{3} \mathrm{O}_{8}\right)$, a hole-doped cuprate $\left(\mathrm{La}_{1.85} \mathrm{Sr}_{0.15} \mathrm{CuO}_{4}\right)$, and a hole-doped IL nickelate $\left(\mathrm{Nd}_{0.775} \mathrm{Sr}_{0.225} \mathrm{NiO}_{2}\right)$. (D) $\mathrm{RIXS}$ intensity map of $\mathrm{NaNiO}_{2}$ measured as a function of incident photon energy across the $\mathrm{Ni} L_{3}$-edge. (E) Representative RIXS spectra of $\mathrm{LaNiO}_{3}$, LaNiO $\mathrm{Na}_{2}$, and $\mathrm{NaNiO}_{2}$. Black arrows highlight the $0.6 \mathrm{eV}$ features of $\mathrm{LaNiO}_{2}$ and $\mathrm{NdNiO}_{2}$. (F) Calculated RIXS map and XAS (solid black line) of $\mathrm{LaNiO}_{2}$ for a $3 d^{9}+3 d^{8} R$ ground state, with $R$ denoting a charge-transfer to the La cation. The dashed box highlights the same feature as the box in panel D. Panels adapted from Refs. [49, 64].

lower-energy peak (Figure 1C) that is associated with transitions into the doped hole levels [70,71]. These hole-states constitute Zhang-Rice singlets (ZRS), which are plaquettes of two doped holes and four $\mathrm{O}$ atoms in square planar coordination around a $\mathrm{Cu}$ atom, playing the same role as a fully occupied or empty site in an effective single-band Hubbard model [72]. Notably, a recent scanning transmission electron microscopy electron-energy loss spectroscopy (STEM-EELS) study suggested that a similar ZRS peak emerges in IL nickelates upon hole-doping [65], although it carries significantly less spectral weight (Figure 1C). Overall, there is good evidence for reduced oxygen hybridization in nickelates compared to cuprates, which likely comes from an enhanced $\Delta$ value. Direct and quantivative determination of $\Delta$ and $U$ represents an important issue for future soft $\mathrm{x}$-ray studies.

Further insights into the electronic structure can be obtained from $\mathrm{Ni} L$-edge XAS, corresponding to $2 p$-3d multiplet transitions, reflecting the valence configuration of the $\mathrm{Ni}$ ions. In the cases of $\mathrm{NiO}$ and perovskite $R E \mathrm{NiO}_{3}\left(2 p^{6} 3 d^{8}-2 p^{5} 3 d^{9}\right.$ and $2 p^{6} 3 d^{8} \underline{L}^{n}-2 p^{5} 3 d^{9} \underline{L}^{n}$ transitions, respectively) distinct multi-peak structures emerge across the $L_{3}$-edge (Figure 1B) $[28,64,73]$. Conversely, the line shapes of the IL nickelates $\mathrm{LaNiO}_{2}$ and especially of $\mathrm{NdNiO}_{2}$ (Figure 1B) resemble rather the singlepeak XAS spectrum of IL cuprates with only one possible final
XAS state $\left(2 p^{6} 3 d^{9}-2 p^{5} 3 d^{10}\right.$ transition). In more detail, the $L_{3}$-edge XAS of $\mathrm{LaNiO}_{2}$ and $\mathrm{NdNiO}_{2}$ is dominated by a main peak A (Figure 1B), while $\mathrm{LaNiO}_{2}$ shows an additional minor low energy shoulder $\mathrm{A}^{\prime}$ at slightly lower energies. ${ }^{1}$ Figure 1D displays the RIXS intensity map of $\mathrm{NdNiO}_{2}$ as a function of the incident photon energy and Figure 1E shows RIXS spectra for selected incident energies. Importantly, the RIXS spectra of $\mathrm{LaNiO}_{2}$ and $\mathrm{NdNiO}_{2}$ exhibit a distinct feature around $0.6 \mathrm{eV}$ energy loss (Figures 1D,E), which is visible in the RIXS spectra with incident energies coinciding with the XAS peak A (A'). Furthermore, this feature emerges exclusively in the IL compounds and not the perovskite nickelate $\mathrm{LaNiO}_{3}$ (Figure 1E). Using exact diagonalization, the general XAS and RIXS features can be reproduced (Figure 1F) and the $0.6 \mathrm{eV}$ feature can be assigned to the hybridization between the $\mathrm{Ni}$

\footnotetext{
${ }^{1}$ The $\mathrm{A}^{\prime}$ feature in XAS is only visible in $\mathrm{LaNiO}_{2}$ films without a $\mathrm{SrTiO}_{3}$ (STO) capping layer. Based on our recent measurements on $\mathrm{LaNiO}_{2}$ films with a STO capping layer, the $\mathrm{A}^{\prime}$ feature, which arises from the resonance of the $\sim 0.6 \mathrm{eV}$ feature in the RIXS map, coincides with the main XAS peak and becomes invisible. In other words, the XAS of La- and Nd-based infinite layer nickelates (with a STO capping layer) are essentially the same
} 


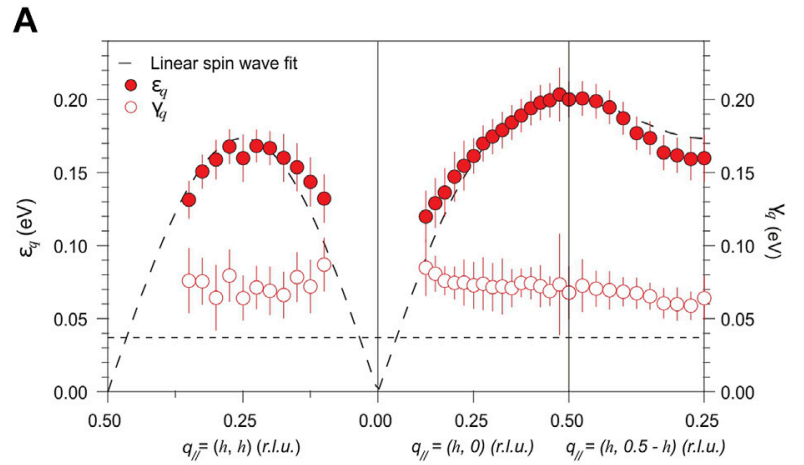

C

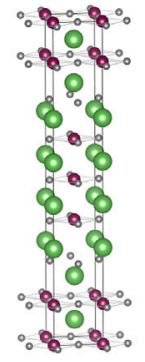

D

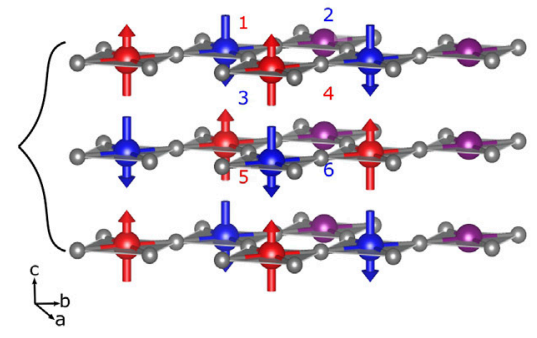

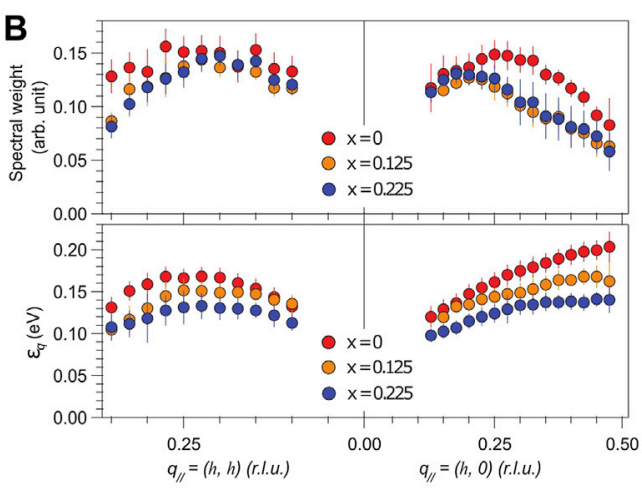

E

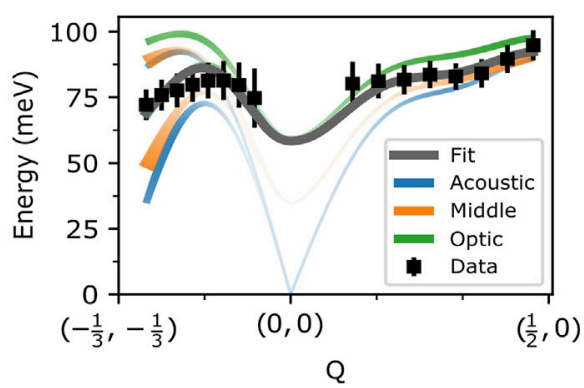

FIGURE 2 | Magnetic excitations in nickelates. (A) Magnetic excitations in $\mathrm{NdNiO}_{2}$. Solid and open symbols are mode energy and the damping parameters, respectively, extracted from fitting RIXS spectra to a damped harmonic oscillators (DHO). The dashed curve is the linear spin wave fit to the extracted energy-momentum dispersion. The horizontal dashed line represents the energy resolution of the RIXS measurement. (B) Doping dependence of the spectral weight (upper) and mode energy (lower) deduced from the $\mathrm{DHO}$ fitting. (C) The structural unit cell of $\mathrm{La}_{4} \mathrm{Ni}_{3} \mathrm{O}_{8}$ with Ni/O/La atoms shown as purple/gray/green spheres. (D) The electronically active TL nickel-oxide plane structures in $\mathrm{La}_{4} \mathrm{Ni}_{3} \mathrm{O}_{8}$, showing the diagonal stripe-ordered state [47]. Ni sites with additional hole character are in purple, whereas spinful Ni up (down) sites are depicted in red (blue). (E) Measured magnetic excitations in $\mathrm{La}_{4} \mathrm{Ni}_{3} \mathrm{O}_{8}$. Black squares are the extracted energies of the magnetic excitations. The dark gray line is the fit to the experimental dispersion, which is composed of three modes plotted in blue, orange, and green, respectively. The doubling of the modes from $\left(-\frac{1}{3},-\frac{1}{3}\right)$ to $(0,0)$ arises from magnetic twinning and the line thickness reflects the predicted intensity of the modes. Panels adapted from Refs. [49, 66].

$3 d_{x^{2}-y^{2}}$ and La $5 d$ orbitals, involving a charge-transfer from Ni to the $R E$ cation [64]. From LDA $+U$ it is found that a Fermi pocket of mainly La $5 d$ character forms near the $\Gamma$ point, which is quite extended and three-dimensional. On the other hand, the $\mathrm{Ni}$ $3 d_{x^{2}-y^{2}}$ states in the $\mathrm{NiO}_{2}$ planes are quasi-2D and strongly correlated [64, 74]. Nevertheless, the relevance of the rareearth $5 d$ bands for the low-energy physics of IL nickelates is still under debate and proposals range from effective single-band to multi-orbital models, including various $\mathrm{Ni} 3 d$ and rare-earth $5 d$ as well as interstitial orbitals [62, 63, 75-89]. Hence, future experiments probing the Fermi surface topology, such as angle resolved photoemission (ARPES) and quantum oscillation measurements, are highly desirable.

\section{MAGNETIC CORRELATIONS}

\subsection{Magnetic Excitations in Infinite-Layer Nickelates}

Despite of the involvement of rare-earth $5 d$ states, the fact that the electronic structures of the $\mathrm{Ni} 3 d$ states resemble a cuprate-like $3 d^{9}$ system raises a curious question: whether the Mott-physics, a key ingredient in the cuprate phenomenology [90], also play an important role in sculpting the electronic structures in IL nickelates. Since a strong AFM interaction is a consequence of Mott physics due to strong onsite Coulomb interaction, information about the magnetic structures in IL nickelate is imperative to gain further insight into this issue. Early investigations of bulk polycrystalline $\mathrm{LaNiO}_{2}$ and $\mathrm{NdNiO}_{2}$ found no evidence of AFM order [59, 60], which appeared to suggest a significantly weaker magnetic interaction than in cuprates. On a different ground, theories have been debating the energy scale of magnetic interactions in the IL nickelates. Some theories predict a small AFM interaction ( an order of magnitude smaller than that of cuprates) because of the larger charge transfer energy $\Delta$ [91-94]. Conversely, other theories argue that the magnetic interactions are comparable to those in cuprates [74, 92, 95]. Experimental information about magnetic excitations is crucial to clarify this important issue.

Recently, magnetic excitations in $\mathrm{Nd}_{1-x} \mathrm{Sr}_{x} \mathrm{NiO}_{2}$ have been revealed using RIXS at the $\mathrm{Ni} L_{3}$-edge [66]. As shown in Figure 2A, a branch of dispersive magnetic excitations has been observed in $\mathrm{NdNiO}_{2}$, whose energy-momentum dispersion resembles the spin wave excitations of AFM coupled spins in a square lattice. Importantly, the bandwidth of the magnetic excitations is approximately $200 \mathrm{meV}$, 
corresponding to a nearest neighbor spin interaction $J_{1} \sim 65 \mathrm{meV}$. This is about half of the $J_{1}$ in cuprate superconductors and similar to that in the TL nickelates [49], but is notably higher than in the stripe-ordered single-layer $(n=1)$ nickelates [25-27], perovskite nickelates $(n=\infty)$ [96], and cubic NiO [97, 98]. Therefore, the observation of the high energy scale of $J_{1}$ in IL nickelates confirms the presence of a strong onsite Coulomb interaction, indicating that the strong correlation effect associated with the Mott-physics is likely also at play in the nickelate superconductors. Notably, distinct from the sharp magnetic modes observed in undoped cuprates, the magnetic excitations in the undoped parent compound of IL nickelates are damped, which is likely due to the coupling to the metallic $\mathrm{Nd} 5 d$ states.

Upon hole doping, the magnetic excitations become less dispersive as a function of momentum and significantly damped. By fitting the spectrum to a damped harmonic oscillator function, it is found that mode energies soften accompanied by slightly reduced spectral weight (Figure 2B). The observed doping dependence is consistent with spin dilution in a Mott insulator. This is in fact different from those observed in cuprates, in which the mode energy and spectral weight do not decrease with increasing doping [99]. The doping dependence of the magnetic excitations in cuprates has been attributed to the longer-range charge dynamics emergent with increasing hole doping, for example, the three site terms in a Hubbard model $[100,101]$. Such dynamics appear to be less prominent in the doped IL nickelates, likely due to the larger charge transfer energy $\Delta$ and the presence of the rare-earth $5 d$ metallic state, calling for further investigation.

We note the next nearest-neighbour exchange interaction $J_{2}$ extracted from the magnetic excitations dispersion possesses an opposite sign to the nearest neighbor $J_{1}$, which should favor the formation of AFM ordering at $(0.5,0.5)$. Unfortunately, RIXS at the $\mathrm{Ni} L_{3}$-edge cannot reach $(0.5,0.5)$ due to insufficient momentum transfer of the photons, preventing a direct scrutinization on the putative AFM order. Notably, recent susceptibility measurement on bulk powder samples indicated spin glass behaviors, but signatures of an AFM phase transition were still not observed. Thus, it would be interesting to investigating why IL nickelates are a failed AFM. However, one should be cautious about the difference between bulk and thin film samples, as well as the disorders in both types of materials, which were significantly reduced over time along with the optimization of material synthesis protocols.

Interestingly, the IL nickelates add one more case in which the magnetic correlations are in proximity to superconductivity in the phase diagram, similarly to a number of unconventional superconductors, such as cuprates, iron-based superconductors, and heavy fermion superconductors [102]. It might be tempting to attribute magnetic fluctuations as a candidate mechanism of superconductivity. However, among these superconducting compounds, including the nickelate superconductors, there appears no clear correlation between the energy scale of the magnetic excitation and the superconducting transition temperature, casting doubt on this notion. In any case, the relationship between magnetic fluctuations and the superconductivity remains an important issue in nickelate superconductors.

\subsection{Magnetic Excitations in Trilayer Nickelates}

In parallel with the measurement of magnetic excitations in the IL material $\mathrm{Nd}_{1-x} \mathrm{Sr}_{x} \mathrm{NiO}_{2}$, magnetic excitations were also measured in the TL materials $\mathrm{La}_{4} \mathrm{Ni}_{3} \mathrm{O}_{8}$ and $\mathrm{Pr}_{4} \mathrm{Ni}_{3} \mathrm{O}_{8}$ [49]. The crystal structure of $\mathrm{La}_{4} \mathrm{Ni}_{3} \mathrm{O}_{8}$ is shown in Figure 2C. This material has some features that indicate that it might be especially promising as a cuprate analog. The rock salt $R E-\mathrm{O}$ layers present in its structure make it more two-dimensional than IL compounds and the rare-earth orbitals that are populated in IL are predicted to have a less significant role in TL systems [48, 103, 104]. As explained in the introduction, this compound is naturally selfdoped and has a nominal hole concentration of $1 / 3$. A disadvantage of the $\mathrm{La}_{4} \mathrm{Ni}_{3} \mathrm{O}_{8}$ series is that they have, to date, proven difficult to chemically dope. Like cuprates, and some other complex oxides, $\mathrm{La}_{4} \mathrm{Ni}_{3} \mathrm{O}_{8}$ has charge and spin order $[47,105]$. This structure, illustrated in Figure 2D, features diagonal rows of $\mathrm{Ni}$ sites with enhanced hole character and neighboring diagonal stripes of up and down spin-ordered sites with reduced hole character. The overall magnetic dispersion, measured with $\mathrm{Ni} L_{3}$ edge RIXS, is plotted in Figure 2E and features a bandwidth of $80 \mathrm{meV}$ with a downturn near $\left(-\frac{1}{3},-\frac{1}{3}\right)$, which is the charge and spin stripe-ordering wavevector. $\mathrm{La}_{4} \mathrm{Ni}_{3} \mathrm{O}_{8}$ was modeled by solving a Heisenberg Hamiltonian which accounts for stripes, and assumes complete charge disproportionation into $d^{9}$ and $d^{8}$ sites, similar to prior studies of other stripe-ordered cuprates and nickelates $[18,106]$. This includes $J_{1}$, which connects sites 1 and 2 in Figure 2D, $J_{3}$, which couples atom 2, through the purple doped site, to atom 1 in the next unit cell and $J_{z}$, which reflects interactions along the $c$-axis, for example, site 1 to site 3 . The diagonal $J_{2}$ interaction that was included in the analysis of $\mathrm{NdNiO}_{2}$ is not expected to be important here, as it couples to the sites with an enhanced hole character and which would be spinless when hosting an extra hole $(S=0)$ [48]. Solving this Hamiltonian in the spin-wave approximation yields three modes. Since these modes could not be resolved separately, the RIXS intensity of these modes was computed and summed to predict the intensity of the magnetic feature in RIXS. Values of $J_{1}=69(4)$ $\mathrm{meV}$ and $J_{3}=17(4) \mathrm{meV}$ were obtained by fitting a Hamiltonian of this type. ${ }^{2}$ Similar to the $\mathrm{Nd}_{1-x} \mathrm{Sr}_{x} \mathrm{NiO}_{2}$ case, the effects of $J_{z}$ were too small to be constrained by the experiment, so the theoretical value of $J_{z}=13.6 \mathrm{meV}$ was used. Very similar dispersions were found in $\operatorname{Pr}_{4} \mathrm{Ni}_{3} \mathrm{O}_{8}$, which may imply that dynamical stripes exist in this compound even though long range stripe order has not been detected, as has been suggested independently in muon spin rotation studies [107].

The leading value of $J_{1}=69(4) \mathrm{meV}$ in $\mathrm{La}_{4} \mathrm{Ni}_{3} \mathrm{O}_{8}$ is strikingly close the $65(1) \mathrm{meV}$ value obtained for $\mathrm{NdNiO}_{2}$. It should be noted that this similar value arises from a much smaller magnetic

${ }^{2}$ The notation used here was been modified from the original work of Ref. [49] to facilitate comparison with Ref. [66]. 
bandwidth, as within the stripe-ordered state, each $\mathrm{Ni}$ will have only two magnetic neighbors. An approximate extrapolation of the magnetic dispersion of $\mathrm{Nd}_{1-x} \mathrm{Sr}_{x} \mathrm{NiO}_{2}$ to a doping of $x \sim 1 / 3$ implies that it would have a bandwidth comparable to $\mathrm{La}_{4} \mathrm{Ni}_{3} \mathrm{O}_{8}$ at this doping; yet, whether stripe order or fluctuations exist in the IL nickelates, like those found in the TL nickelates, remains an important open question. Overall, this suggests that the local correlated physics in these reduced RP cousins is very similar provided they are compared at the same effective doping, although their precise low-energy ground states might be more different.

\section{CONCLUSION}

In summary, soft $\mathrm{x}$-ray spectroscopic studies have provided valuable insights into the physics of RP-phase and RP-derived nickelates. Nevertheless, for low-valence nickelates there is still limited consensus on the essential ingredients of their electronic structure. Along these lines, we anticipate that advances in sample synthesis and the application of complementary experimental techniques, including ARPES and quantum oscillation measurements, will be helpful. Moreover, the role of disorder and capping layers, as well as the apparent differences between film and bulk samples need further clarification. These insights could point the way towards improved low-valance nickelate superconductors, including multilayer systems [108]. Finally, a pertinent question is whether suitable sample preparation allows to realize other cuprate-typical ground states in nickelates, such as

\section{REFERENCES}

1. Keimer B, Kivelson SA, Norman MR, Uchida S, Zaanen J. From Quantum Matter to High-Temperature Superconductivity in Copper Oxides. Nature (2015) 518:179-86. doi:10.1038/nature14165

2. Lee PA, Nagaosa N, Wen XG. Doping a Mott Insulator: Physics of HighTemperature Superconductivity. Rev Mod Phys (2006) 78:17-85. doi:10.1103/ RevModPhys.78.17

3. Armitage NP, Fournier P, Greene RL. Progress and Perspectives on ElectronDoped Cuprates. Rev Mod Phys (2010) 82:2421-87. doi:10.1103/ RevModPhys.82.2421

4. Damascelli A, Hussain Z, Shen ZX. Angle-resolved Photoemission Studies of the Cuprate Superconductors. Rev Mod Phys (2003) 75:473-541. doi:10.1103/ RevModPhys.75.473

5. Fink J, Knupfer M, Atzkern S, Golden M. Electronic Correlations in Solids, Studied Using Electron Energy-Loss Spectroscopy. J Electron Spectros Relat Phenomena (2001) 117-118:287-309. doi:10.1016/S0368-2048(01)00254-7

6. Fischer O, Kugler M, Maggio-Aprile I, Berthod C, Renner C. Scanning Tunneling Spectroscopy of High-Temperature Superconductors. Rev Mod Phys (2007) 79:353-419. doi:10.1103/RevModPhys.79.353

7. Basov DN, Timusk T. Electrodynamics of High- $T_{c}$ Superconductors. Rev Mod Phys (2005) 77:721-79. doi:10.1103/RevModPhys.77.721

8. Devereaux TP, Hackl R. Inelastic Light Scattering from Correlated Electrons. Rev Mod Phys (2007) 79:175-233. doi:10.1103/RevModPhys.79.175

9. Fujita M, Hiraka H, Matsuda M, Matsuura M, Tranquada MJ, Wakimoto S, et al. Progress in Neutron Scattering Studies of Spin Excitations in High- $T_{c}$ Cuprates. J Phys Soc Jpn (2012) 81:011007. doi:10.1143/JPSJ.81.011007

10. Miao H, Ishikawa D, Heid R, Le Tacon M, Fabbris G, Meyers D, et al. Incommensurate Phonon Anomaly and the Nature of Charge Density Waves in Cuprates. Phys Rev X (2018) 8:011008. doi:10.1103/PhysRevX.8.011008 antiferromagnetism, pseudogap, as well as nematic, charge, and spin orders.

\section{AUTHOR CONTRIBUTIONS}

The manuscript was written by MH, MD, and W-SL.

\section{FUNDING}

Work at SLAC National Lab was supported by United States Department of Energy, Office of Science, Office of Basic Energy Sciences under Contract No. DE-AC02-76SF00515. Work at Brookhaven National Laboratory was supported by the United States Department of Energy, Office of Science, Office of Basic Energy Sciences. The use of resources at the SIX beamline of the National Synchrotron Light Source II, a United States Department of Energy (DOE) Office of Science User Facility operated for the DOE Office of Science by Brookhaven National Laboratory under Contract No. DE-SC0012704, is acknowledged. The Max Planck Society is acknowledged for funding of the open access fee.

\section{ACKNOWLEDGMENTS}

The authors thank K. Fürsich for useful discussions and comments.

11. Ament LJP, van Veenendaal M, Devereaux TP, Hill JP, van den Brink J. Resonant Inelastic X-ray Scattering Studies of Elementary Excitations. Rev Mod Phys (2011) 83:705-67. doi:10.1103/RevModPhys.83.705

12. Le Tacon M, Ghiringhelli G, Chaloupka J, Sala MM, Hinkov V, Haverkort M, et al. Intense Paramagnon Excitations in a Large Family of High-Temperature Superconductors. Nat Phys (2011) 7:725-30. doi:10.1038/NPHYS2041

13. Dean M, Dellea G, Springell R, Yakhou-Harris F, Kummer K, Brookes N, et al. Persistence of Magnetic Excitations in $\mathrm{La}_{2-x} \mathrm{Sr}_{x} \mathrm{CuO}_{4}$ from the Undoped Insulator to the Heavily Overdoped Non-superconducting Metal. Nat Mater (2013) 12:1019-23. doi:10.1038/NMAT3723

14. Hepting M, Chaix L, Huang E, Fumagalli R, Peng Y, Moritz B, et al. Threedimensional Collective Charge Excitations in Electron-Doped Copper Oxide Superconductors. Nature (2018) 563:374-8. doi:10.1038/s41586-018-0648-3

15. Lin JQ, Yuan J, Jin K, Yin ZP, Li G, Zhou K-J, et al. Doping Evolution of the Charge Excitations and Electron Correlations in Electron-Doped Superconducting $\mathrm{La}_{2-x} \mathrm{Ce}_{x} \mathrm{CuO}_{4}$. Npj Quan Mater (2020) 5:4. doi:10.1038/ s41535-019-0205-9

16. Nag A, Zhu M, Bejas M, Li J, Robarts HC, Yamase H, et al. Detection of Acoustic Plasmons in Hole-Doped Lanthanum and Bismuth Cuprate Superconductors Using Resonant Inelastic X-ray Scattering. Phys Rev Lett (2020) 125:257002. doi:10.1103/PhysRevLett.125.257002

17. Ghiringhelli G, Le Tacon M, Minola M, Blanco-Canosa S, Mazzoli C, Brookes $\mathrm{NB}$, et al. Long-Range Incommensurate Charge Fluctuations in (Y,Nd) $\mathrm{Ba}_{2} \mathrm{Cu}_{3} \mathrm{O}_{6+x}$. Science (2012) 337:821-5. doi:10.1126/science.1223532

18. Miao H, Lorenzana J, Seibold G, Peng YY, Amorese A, Yakhou-Harris F, et al. High-temperature Charge Density Wave Correlations in $\mathrm{La}_{1.875} \mathrm{Ba}_{0.125} \mathrm{CuO}_{4}$ without Spin-Charge Locking. PNAS (2017) 114:12430-5. doi:10.1073/ pnas. 1708549114

19. Chaix L, Ghiringhelli G, Peng Y, Hashimoto M, Moritz B, Kummer K, et al. Dispersive Charge Density Wave Excitations in $\mathrm{Bi}_{2} \mathrm{Sr}_{2} \mathrm{CaCu}_{2} \mathrm{O}_{8+\delta}$. Nat Phys (2017) 13:952-6. doi:10.1038/nphys4157 
20. Miao H, Fumagalli R, Rossi M, Lorenzana J, Seibold G, Yakhou-Harris F, et al. Formation of Incommensurate Charge Density Waves in Cuprates. Phys Rev $X$ (2019) 9:031042. doi:10.1103/PhysRevX.9.031042

21. Lee WS, Zhou KJ, Hepting M, Li J, Nag A, Walters AC, et al. Spectroscopic Fingerprint of Charge Order Melting Driven by Quantum Fluctuations in a Cuprate. Nat Phys (2021) 17:53-7. doi:10.1038/s41567-020-0993-7

22. Norman MR. Materials Design for New Superconductors. Rep Prog Phys (2016) 79:074502. doi:10.1088/0034-4885/79/7/074502

23. Greenblatt M. Ruddlesden-popper $\operatorname{Ln}_{n+1} \mathrm{Ni}_{n} \mathrm{O}_{3 n+1}$ Nickelates: Structure and Properties. Curr Opin Solid State Mater Sci (1997) 2:174-83. doi:10.1016/ S1359-0286(97)80062-9

24. Acrivos J, Lei M, Jiang C, Nguyen H, Metcalf $\mathrm{P}$, Honig J. Paramagnetism, Antiferromagnetism, and Superconductivity in $\mathrm{La}_{2} \mathrm{NiO}_{4}$. J Solid State Chem (1994) 111:343-8. doi:10.1006/jssc.1994.1237

25. Sachan V, Buttrey DJ, Tranquada JM, Lorenzo JE, Shirane G. Charge and Spin Ordering in $\mathrm{La}_{2-x} \mathrm{Sr}_{x} \mathrm{NiO}_{4.00}$ with $\mathrm{x}=0.135$ and 0.20. Phys Rev B (1995) 51:12742-6. doi:10.1103/PhysRevB.51.12742

26. Sugai S, Sato M, Kobayashi T, Akimitsu J, Ito T, Takagi H, et al. High-energy Spin Excitations in the Insulating Phases of High- $T_{c}$ Superconducting Cuprates and $\mathrm{La}_{2} \mathrm{NiO}_{4}$. Phys Rev $B$ (1990) 42:1045-7. doi:10.1103/ PhysRevB.42.1045

27. Fabbris G, Meyers D, Xu L, Katukuri VM, Hozoi L, Liu X, et al. Doping Dependence of Collective Spin and Orbital Excitations in the Spin-1 Quantum Antiferromagnet $\mathrm{La}_{2-x} \mathrm{Sr}_{x} \mathrm{NiO}_{4}$ Observed by X Rays. Phys Rev Lett (2017) 118:156402. doi:10.1103/PhysRevLett.118.156402

28. Bisogni V, Catalano S, Green RJ, Gibert M, Scherwitzl R, Huang Y, et al. Ground-state Oxygen Holes and the Metal-Insulator Transition in the Negative Charge-Transfer Rare-Earth Nickelates. Nat Commun (2016) 7: 13017. doi:10.1038/ncomms 13017

29. Chaloupka J, Khaliullin G. Orbital Order and Possible Superconductivity in $\mathrm{LaNiO}_{3} / \mathrm{LaMO}_{3}$ Superlattices. Phys Rev Lett (2008) 100:016404. doi:10.1103/ PhysRevLett.100.016404

30. Hansmann P, Yang X, Toschi A, Khaliullin G, Andersen OK, Held K. Turning a Nickelate Fermi Surface into a Cupratelike One through Heterostructuring. Phys Rev Lett (2009) 103:016401. doi:10.1103/ PhysRevLett.103.016401

31. Middey S, Chakhalian J, Mahadevan P, Freeland J, Millis A, Sarma D. Physics of Ultrathin Films and Heterostructures of Rare-Earth Nickelates. Annu Rev Mater Res (2016) 46:305-34. doi:10.1146/annurev-matsci070115-032057

32. Catalano S, Gibert M, Fowlie J, Îniguez J, Triscone JM, Kreisel J. Rare-earth Nickelates $\mathrm{RNiO}_{3}$ : Thin Films and Heterostructures. Rep Prog Phys (2018) 81: 046501. doi:10.1088/1361-6633/aaa37a

33. Boris AV, Matiks Y, Benckiser E, Frano A, Popovich P, Hinkov V, et al. Dimensionality Control of Electronic Phase Transitions in Nickel-Oxide Superlattices. Science (2011) 332:937-40. doi:10.1126/science.1202647

34. Benckiser E, Haverkort MW, Brück S, Goering E, Macke S, Frañó A, et al. Orbital Reflectometry of Oxide Heterostructures. Nat Mater (2011) 10: 189-93. doi:10.1038/NMAT2958

35. Liu J, Okamoto S, van Veenendaal M, Kareev M, Gray B, Ryan P, et al. Quantum Confinement of mott Electrons in Ultrathin $\mathrm{LaNiO}_{3} / \mathrm{LaAlO}_{3}$ Superlattices. Phys Rev B (2011) 83:161102. doi:10.1103/PhysRevB.83.161102

36. Frano A, Schierle E, Haverkort MW, Lu Y, Wu M, Blanco-Canosa S, et al. Orbital Control of Noncollinear Magnetic Order in Nickel Oxide Heterostructures. Phys Rev Lett (2013) 111:106804. doi:10.1103/ PhysRevLett.111.106804

37. Hepting M, Minola M, Frano A, Cristiani G, Logvenov G, Schierle E, et al. Tunable Charge and Spin Order in $\mathrm{PrNiO}_{3}$ Thin Films and Superlattices. Phys Rev Lett (2014) 113:227206. doi:10.1103/PhysRevLett.113.227206

38. Gibert M, Viret M, Torres-Pardo A, Piamonteze C, Zubko P, Jaouen N, et al. Interfacial Control of Magnetic Properties at $\mathrm{LaMnO}_{3} / \mathrm{LaNiO}_{3}$ Interfaces. Nano Lett (2015) 15:7355-61. doi:10.1021/acs.nanolett.5b02720

39. Disa AS, Georgescu AB, Hart JL, Kumah DP, Shafer P, Arenholz E, et al. Control of Hidden Ground-State Order in $\mathrm{NdNiO}_{3}$ Superlattices. Phys Rev Mater (2017) 1:024410. doi:10.1103/PhysRevMaterials.1.024410

40. Hepting M, Green RJ, Zhong Z, Bluschke M, Suyolcu YE, Macke S, et al. Complex Magnetic Order in Nickelate Slabs. Nat Phys (2018) 14:1097-102. doi:10.1038/s41567-018-0218-5
41. Fürsich K, Lu Y, Betto D, Bluschke M, Porras I, Schierle E, et al. Resonant Inelastic X-ray Scattering Study of Bond Order and Spin Excitations in Nickelate Thin-Film Structures. Phys Rev B (2019) 99:165124. doi:10.1103/ PhysRevB.99.165124

42. Chakhalian J, Rondinelli JM, Liu J, Gray BA, Kareev M, Moon EJ, et al. Asymmetric Orbital-Lattice Interactions in Ultrathin Correlated Oxide Films. Phys Rev Lett (2011) 107:116805. doi:10.1103/PhysRevLett.107.116805

43. Wu M, Benckiser E, Haverkort MW, Frano A, Lu Y, Nwankwo U, et al. Strain and Composition Dependence of Orbital Polarization in Nickel Oxide Superlattices. Phys Rev B (2013) 88:125124. doi:10.1103/PhysRevB.88.125124

44. Disa AS, Kumah DP, Malashevich A, Chen H, Arena DA, Specht ED, et al. Orbital Engineering in Symmetry-Breaking Polar Heterostructures. Phys Rev Lett (2015) 114:026801. doi:10.1103/PhysRevLett.114.026801

45. Han MJ, Wang X, Marianetti CA, Millis AJ. Dynamical Mean-Field Theory of Nickelate Superlattices. Phys Rev Lett (2011) 107:206804. doi:10.1103/ PhysRevLett.107.206804

46. Fabbris G, Meyers D, Okamoto J, Pelliciari J, Disa AS, Huang Y, et al. Orbital Engineering in Nickelate Heterostructures Driven by Anisotropic Oxygen Hybridization rather Than Orbital Energy Levels. Phys Rev Lett (2016) 117: 147401. doi:10.1103/PhysRevLett.117.147401

47. Zhang J, Chen YS, Phelan D, Zheng H, Norman MR, Mitchell JF. Stacked Charge Stripes in the quasi-2D Trilayer Nickelate $\mathrm{La}_{4} \mathrm{Ni}_{3} \mathrm{O}_{8}$. PNAS (2016) 113:8945-50. doi:10.1073/pnas.1606637113

48. Zhang J, Botana AS, Freeland JW, Phelan D, Zheng H, Pardo V, et al. Large Orbital Polarization in a Metallic Square-Planar Nickelate. Nat Phys (2017) 13:864-9. doi:10.1038/nphys4149

49. Lin JQ, Villar Arribi P, Fabbris G, Botana AS, Meyers D, Miao H, et al. Strong Superexchange in a $d^{9-\delta}$ Nickelate Revealed by Resonant Inelastic X-ray Scattering. Phys Rev Lett (2021) 126:087001. doi:10.1103/ PhysRevLett.126.087001

50. Botana AS, Pardo V, Norman MR. Electron Doped Layered Nickelates: Spanning the Phase Diagram of the Cuprates. Phys Rev Mater (2017) 1: 021801. doi:10.1103/PhysRevMaterials.1.021801

51. Nica EM, Krishna J, Yu R, Si Q, Botana AS, Erten O. Theoretical Investigation of Superconductivity in Trilayer Square-Planar Nickelates. Phys Rev B (2020) 102:020504. doi:10.1103/PhysRevB.102.020504

52. Li D, Lee K, Wang BY, Osada M, Crossley S, Lee HR, et al. Superconductivity in an Infinite-Layer Nickelate. Nature (2019) 572:624-7. doi:10.1038/s41586019-1496-5

53. Zeng S, Tang CS, Yin X, Li C, Li M, Huang Z, et al. Phase Diagram and Superconducting Dome of Infinite-Layer $\mathrm{Nd}_{1-x} \mathrm{Sr}_{x} \mathrm{NiO}_{2}$ Thin Films. Phys Rev Lett (2020) 125:147003. doi:10.1103/physrevlett.125.147003

54. Li D, Wang BY, Lee K, Harvey SP, Osada M, Goodge BH, et al. Superconducting Dome in $\mathrm{Nd}_{1-x} \mathrm{Sr}_{x} \mathrm{NiO}_{2}$ Infinite Layer Films. Phys Rev Lett (2020) 125:027001. doi:10.1103/PhysRevLett.125.027001

55. Lee K, Goodge BH, Li D, Osada M, Wang BY, Cui Y, et al. Aspects of the Synthesis of Thin Film Superconducting Infinite-Layer Nickelates. APL Mater (2020) 8:041107. doi:10.1063/5.0005103

56. Osada M, Wang BY, Goodge BH, Lee K, Yoon H, Sakuma K, et al. A Superconducting Praseodymium Nickelate with Infinite Layer Structure. Nano Lett (2020) 20:5735-40. doi:10.1021/acs.nanolett.0c01392

57. Li Y, Sun W, Yang J, Cai X, Guo W, Gu Z, et al. Impact of Cation Stoichiometry on the Crystalline Structure and Superconductivity in Nickelates. Front Phys (2021) 9:443. doi:10.3389/fphy.2021.719534

58. Osada M, Wang BY, Goodge BH, Harvey SP, Lee K, Li D, et al. Nickelate Superconductivity without Rare-Earth Magnetism: ( $\mathrm{La}, \mathrm{Sr}) \mathrm{NiO}_{2}$. Adv Mater (2021) 33:2104083. doi:10.1002/adma.202104083

59. Hayward MA, Green MA, Rosseinsky MJ, Sloan J. Sodium Hydride as a Powerful Reducing Agent for Topotactic Oxide Deintercalation: Synthesis and Characterization of the Nickel(i) Oxide $\mathrm{LaNiO}_{2}$. J Am Chem Soc (1999) 121:8843-54. doi:10.1021/ja991573i

60. Hayward M, Rosseinsky M. Synthesis of the Infinite Layer Ni(i) Phase $\mathrm{NdNiO}_{2+x}$ by Low Temperature Reduction of $\mathrm{NdNiO}_{3}$ with Sodium Hydride. Solid State Sci (2003) 5:839-50. doi:10.1016/S1293-2558(03) 00111-0

61. Ikeda A, Krockenberger Y, Irie H, Naito M, Yamamoto H. Direct Observation of Infinite $\mathrm{NiO}_{2}$ Planes in $\mathrm{LaNiO}_{2}$ Films. Appl Phys Express (2016) 9:061101. doi:10.7567/apex.9.061101 
62. Anisimov VI, Bukhvalov D, Rice TM. Electronic Structure of Possible Nickelate Analogs to the Cuprates. Phys Rev B (1999) 59:7901-6. doi:10.1103/physrevb.59.7901

63. Lee KW, Pickett WE. Infinite-layer $\mathrm{LaNiO}_{2}: \mathrm{Ni}^{1+}$ Is $\mathrm{Not}^{\mathrm{Cu}}{ }^{2+}$. Phys Rev $B$ (2004) 70:165109. doi:10.1103/physrevb.70.165109

64. Hepting M, Li D, Jia CJ, Lu H, Paris E, Tseng Y, et al. Electronic Structure of the Parent Compound of Superconducting Infinite-Layer Nickelates. Nat Mater (2020) 19:381-5. doi:10.1038/s41563-019-0585-z

65. Goodge BH, Li D, Lee K, Osada M, Wang BY, Sawatzky GA, et al. Doping Evolution of the Mott-Hubbard Landscape in Infinite-Layer Nickelates. PNAS (2021) 118:e2007683118. doi:10.1073/pnas.2007683118

66. Lu H, Rossi M, Nag A, Osada M, Li DF, Lee K, et al. Magnetic Excitations in Infinite-Layer Nickelates. Science (2021) 373:213-6. doi:10.1126/ science.abd 7726

67. Abbate M, Zampieri G, Prado F, Caneiro A, Gonzalez-Calbet JM, Vallet-Regi M. Electronic Structure and Metal-Insulator Transition in $\mathrm{LaNiO}_{3-\delta}$. Phys Rev B (2002) 65:155101. doi:10.1103/physrevb.65.155101

68. Kuiper P, Kruizinga G, Ghijsen J, Sawatzky GA, Verweij H. Character of Holes in $\mathrm{Li}_{x} \mathrm{Ni}_{1-x} \mathrm{O}$ and Their Magnetic Behavior. Phys Rev Lett (1989) 62: 221-4. doi:10.1103/PhysRevLett.62.221

69. Zaanen J, Sawatzky GA, Allen JW. Band Gaps and Electronic Structure of Transition-Metal Compounds. Phys Rev Lett (1985) 55:418-21. doi:10.1103/ PhysRevLett.55.418

70. Chen CT, Sette F, Ma Y, Hybertsen MS, Stechel EB, Foulkes WMC, et al. Electronic States in $\mathrm{La}_{2-x} \mathrm{Sr}_{x} \mathrm{CuO}_{4+\delta}$ Probed by Soft-X-ray Absorption. Phys Rev Lett (1991) 66:104-7. doi:10.1103/PhysRevLett.66.104

71. Abbamonte P, Rusydi A, Smadici S, Gu GD, Sawatzky GA, Feng DL. Spatially Modulated 'Mottness' in $\mathrm{La}_{2-x} \mathrm{Ba}_{x} \mathrm{CuO}_{4}$. Nat Phys (2005) 1:155-8. doi:10.1038/nphys178

72. Zhang FC, Rice TM. Effective Hamiltonian for the Superconducting $\mathrm{Cu}$ Oxides. Phys Rev B (1988) 37:3759-61. doi:10.1103/PhysRevB.37.3759

73. Green RJ, Haverkort MW, Sawatzky GA. Bond Disproportionation and Dynamical Charge Fluctuations in the Perovskite Rare-Earth Nickelates. Phys Rev B (2016) 94:195127. doi:10.1103/PhysRevB.94.195127

74. Been E, Lee WS, Hwang HY, Cui Y, Zaanen J, Devereaux T, et al. Electronic Structure Trends across the Rare-Earth Series in Superconducting Infinite-Layer Nickelates. Phys Rev X (2021) 11:011050. doi:10.1103/physrevx.11.011050

75. Nomura Y, Hirayama M, Tadano T, Yoshimoto Y, Nakamura K, Arita R. Formation of a Two-Dimensional Single-Component Correlated Electron System and Band Engineering in the Nickelate Superconductor $\mathrm{NdNio}_{2}$. Phys Rev B (2019) 100:205138. doi:10.1103/PhysRevB.100.205138

76. Botana A, Norman M. Similarities and Differences between $\mathrm{LaNiO}_{2}$ and $\mathrm{CaCuO}_{2}$ and Implications for Superconductivity. Phys Rev X (2020) 10: 011024. doi:10.1103/physrevx.10.011024

77. Kitatani M, Si L, Janson O, Arita R, Zhong Z, Held K. Nickelate Superconductors-A Renaissance of the One-Band Hubbard Model. Npj Quan Mater (2020) 5:59. doi:10.1038/s41535-020-00260-y

78. Wu X, Di Sante D, Schwemmer T, Hanke W, Hwang HY, Raghu S, et al. Robust $d_{x^{2}-y^{2}}$-wave Superconductivity of Infinite-Layer Nickelates. Phys Rev B (2020) 101:060504. doi:10.1103/PhysRevB.101.060504

79. Zhang H, Jin L, Wang S, Xi B, Shi X, Ye F, et al. Effective Hamiltonian for Nickelate Oxides $\mathrm{Nd}_{1-x} \mathrm{Sr}_{x} \mathrm{NiO}_{2}$. Phys Rev Res (2020) 2:013214. doi:10.1103/ PhysRevResearch.2.013214

80. Sakakibara H, Usui H, Suzuki K, Kotani T, Aoki H, Kuroki K. Model Construction and a Possibility of Cupratelike Pairing in a New $d^{9}$ Nickelate Superconductor (Nd, Sr) $\mathrm{NiO}_{2}$. Phys Rev Lett (2020) 125:077003. doi:10.1103/PhysRevLett.125.077003

81. Lechermann F Late Transition Metal Oxides with Infinite-Layer Structure: Nickelates versus Cuprates. Phys Rev B (2020) 101:081110. doi:10.1103/ PhysRevB.101.081110

82. Wang Y, Kang CJ, Miao H, Kotliar G. Hund's Metal Physics: From $\mathrm{SrNiO}_{2}$ to $\mathrm{LaNiO}_{2}$. Phys Rev B (2020) 102:161118. doi:10.1103/PhysRevB.102.161118

83. Werner P, Hoshino S. Nickelate Superconductors: Multiorbital Nature and Spin Freezing. Phys Rev B (2020) 101:041104. doi:10.1103/ PhysRevB.101.041104

84. Adhikary P, Bandyopadhyay S, Das T, Dasgupta I, Saha-Dasgupta T. Orbitalselective Superconductivity in a Two-Band Model of Infinite-Layer Nickelates. Phys Rev B (2020) 102:100501. doi:10.1103/PhysRevB.102.100501
85. Karp J, Botana AS, Norman MR, Park H, Zingl M, Millis A. Many-body Electronic Structure of $\mathrm{NdNiO}_{2}$ and $\mathrm{CaCuO}_{2}$. Phys Rev X (2020) 10:021061. doi:10.1103/PhysRevX.10.021061

86. Liu Z, Xu C, Cao C, Zhu W, Wang ZF, Yang J. Doping Dependence of Electronic Structure of Infinite-Layer $\mathrm{NdNiO}_{2}$. Phys Rev B (2021) 103:045103. doi:10.1103/PhysRevB.103.045103

87. Wan X, Ivanov V, Resta G, Leonov I, Savrasov SY. Exchange Interactions and Sensitivity of the Ni Two-Hole Spin State to Hund's Coupling in Doped $\mathrm{NdNiO}_{2}$. Phys Rev $B$ (2021) 103:075123. doi:10.1103/ PhysRevB.103.075123

88. Lang ZJ, Jiang R, Ku W. Strongly Correlated Doped Hole Carriers in the Superconducting Nickelates: Their Location, Local many-body State, and Low-Energy Effective Hamiltonian. Phys Rev B (2021) 103:L180502. doi:10.1103/PhysRevB.103.L180502

89. Higashi K, Winder M, Kuneš J, Hariki A. Core-level X-ray Spectroscopy of Infinite-Layer Nickelate: LDA + DMFT Study. Phys Rev X (2021) 11:041009. doi:10.1103/PhysRevX.11.041009

90. Phillips P. Mottness. Ann Phys (N Y.) (2006) 321:1634-50. doi:10.1016/ j.aop.2006.04.003

91. Jiang M, Berciu M, Sawatzky GA. Critical Nature of the Ni Spin State in Doped $\mathrm{NdNiO}_{2}$. Phys Rev Lett (2020) 124:207004. doi:10.1103/ PhysRevLett.124.207004

92. Zhang GM, Yang Y, Zhang FC. Self-doped mott Insulator for Parent Compounds of Nickelate Superconductors. Phys Rev B (2020) 101:020501. doi:10.1103/PhysRevB.101.020501

93. Hu LH, Wu C. Two-band Model for Magnetism and Superconductivity in Nickelates. Phys Rev Res (2019) 1:032046. doi:10.1103/ PhysRevResearch.1.032046

94. Liu Z, Ren Z, Zhu W, Wang Z, Yang J. Electronic and Magnetic Structure of Infinite-Layer $\mathrm{NdNiO}_{2}$ : Trace of Antiferromagnetic Metal. Npj Quan Mater (2020) 5:31. doi:10.1038/s41535-020-0229-1

95. Katukuri VM, Bogdanov NA, Weser O, van den Brink J, Alavi A. Electronic Correlations and Magnetic Interactions in Infinite-Layer $\mathrm{NdNiO}_{2}$. Phys Rev $B$ (2020) 102:241112. doi:10.1103/PhysRevB.102.241112

96. Lu Y, Betto D, Fürsich K, Suzuki H, Kim HH, Cristiani G, et al. Site-selective Probe of Magnetic Excitations in Rare-Earth Nickelates Using Resonant Inelastic X-ray Scattering. Phys Rev X (2018) 8:031014. doi:10.1103/ PhysRevX.8.031014

97. Hutchings MT, Samuelsen EJ. Measurement of Spin-Wave Dispersion in $\mathrm{NiO}$ by Inelastic Neutron Scattering and its Relation to Magnetic Properties. Phys Rev B (1972) 6:3447-61. doi:10.1103/PhysRevB.6.3447

98. Ghiringhelli G, Piazzalunga A, Dallera C, Schmitt T, Strocov VN, Schlappa J, et al. Observation of Two Nondispersive Magnetic Excitations in NiO by Resonant Inelastic Soft-X-Ray Scattering. Phys Rev Lett (2009) 102:027401. doi:10.1103/PhysRevLett.102.027401

99. Peng YY, Huang EW, Fumagalli R, Minola M, Wang Y, Sun X, et al. Dispersion, Damping, and Intensity of Spin Excitations in the Monolayer $(\mathrm{Bi}, \mathrm{Pb})_{2}(\mathrm{Sr}, \mathrm{La})_{2} \mathrm{CuO}_{6}+\delta$ Cuprate Superconductor Family. Phys Rev B (2018) 98:144507. doi:10.1103/PhysRevB.98.144507

100. Jia CJ, Nowadnick EA, Wohlfeld K, Kung YF, Chen CC, Johnston S, et al. Persistent Spin Excitations in Doped Antiferromagnets Revealed by Resonant Inelastic Light Scattering. Nat Commun (2014) 5:3314. doi:10.1038/ ncomms 4314

101. Bala J, Oleś AM, Zaanen J. Spin Polarons in the T-T'-J Model. Phys Rev B (1995) 52:4597-606. doi:10.1103/PhysRevB.52.4597

102. Scalapino DJ. A Common Thread: The Pairing Interaction for Unconventional Superconductors. Rev Mod Phys (2012) 84:1383-417. doi:10.1103/RevModPhys.84.1383

103. Poltavets VV, Lokshin KA, Nevidomskyy AH, Croft M, Tyson TA, Hadermann J, et al. Bulk Magnetic Order in a Two-Dimensional $\mathrm{Ni}^{1+}$ / $\mathrm{Ni}^{2+}\left(d^{9} / d^{8}\right)$ Nickelate, Isoelectronic with Superconducting Cuprates. Phys Rev Lett (2010) 104:206403. doi:10.1103/PhysRevLett.104.206403

104. Botana AS, Pardo V, Pickett WE, Norman MR. Charge Ordering in $\mathrm{Ni}^{1+} / \mathrm{Ni}^{2+}$ Nickelates: $\mathrm{La}_{4} \mathrm{Ni}_{3} \mathrm{O}_{8}$ and $\mathrm{La}_{3} \mathrm{Ni}_{2} \mathrm{O}_{6}$. Phys Rev $B$ (2016) 94:081105. doi:10.1103/PhysRevB.94.081105

105. Zhang J, Pajerowski DM, Botana AS, Zheng H, Harriger L, Rodriguez-Rivera J, et al. Spin Stripe Order in a Square Planar Trilayer Nickelate. Phys Rev Lett (2019) 122:247201. doi:10.1103/PhysRevLett.122.247201 
106. Carlson EW, Yao DX, Campbell DK. Spin Waves in Striped Phases. Phys Rev $B$ (2004) 70:064505. doi:10.1103/PhysRevB.70.064505

107. Huangfu S, Guguchia Z, Cheptiakov D, Zhang X, Luetkens H, Gawryluk DJ, et al. Short-range Magnetic Interactions and Spin-Glass Behavior in the Quasi-Two-Dimensional Nickelate $\mathrm{Pr}_{4} \mathrm{Ni}_{3} \mathrm{O}_{8}$. Phys Rev B (2020) 102:054423. doi:10.1103/physrevb.102.054423

108. Ortiz RA, Menke H, Misják F, Mantadakis DT, Fürsich K, Schierle E, et al. Superlattice Approach to Doping Infinite-Layer Nickelates. Phys Rev B (2021) 104:165137. doi:10.1103/PhysRevB.104.165137

Conflict of Interest: The authors declare that the research was conducted in the absence of any commercial or financial relationships that could be construed as a potential conflict of interest.
Publisher's Note: All claims expressed in this article are solely those of the authors and do not necessarily represent those of their affiliated organizations, or those of the publisher, the editors and the reviewers. Any product that may be evaluated in this article, or claim that may be made by its manufacturer, is not guaranteed or endorsed by the publisher.

Copyright (c) 2021 Hepting, Dean and Lee. This is an open-access article distributed under the terms of the Creative Commons Attribution License (CC BY). The use, distribution or reproduction in other forums is permitted, provided the original author(s) and the copyright owner(s) are credited and that the original publication in this journal is cited, in accordance with accepted academic practice. No use, distribution or reproduction is permitted which does not comply with these terms. 